\title{
Efficient Micro/Nanoparticle Concentration using Direct Current- Induced Thermal Buoyancy Convection for Multiple Liquid Media
}

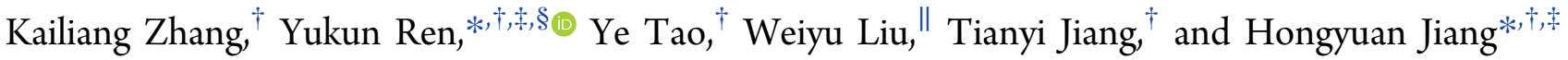 \\ ${ }^{\dagger}$ School of Mechatronics Engineering, Harbin Institute of Technology, Harbin, Heilongiiang 150001, P. R. China \\ ${ }^{\ddagger}$ State Key Laboratory of Robotics and System, Harbin Institute of Technology, Harbin, Heilongjiang 150001, P. R. China \\ ${ }^{\S}$ State Key Laboratory of Nonlinear Mechanics, Chinese Academy of Sciences, Beijing 100190, P. R. China \\ "School of Electronics and Control Engineering, Chang'an University, Xi'an, Shanxi 710064, P. R. China
}

Supporting Information

ABSTRACT: Thermal-based microparticle focusing has recently received increasing attention due to its noninvasive nature and simple manipulation mechanism. However, its further application is limited by current complicated fluid heating systems and low particle focusing velocity. Using simple indium tin oxide-made microheaters, herein we propose a flexible and novel approach for efficient particle focusing based on direct current-induced thermal buoyancy convection. Importantly, for avoiding possible electrochemical reactions on the electrode, the microheaters are isolated from the granular

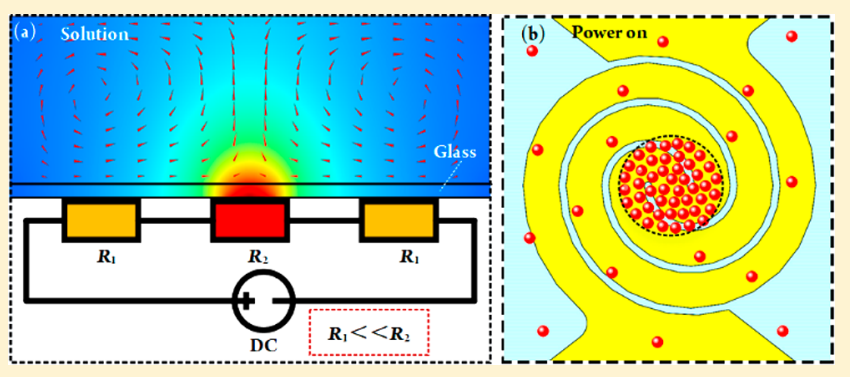
fluids of interest by a thin glass slide. The concentration performance of the designed chip was first demonstrated by statically focusing $4-\mu \mathrm{m}$ silica particles, yeast cells, silica particles in insulating buffer, and $100-\mathrm{nm}$ copper microspheres. Also the trapping of a mixture of 5- $\mu \mathrm{m}$ and $2-\mu \mathrm{m}$ polystyrene microbeads indicated that the chip can either simultaneously concentrate two kinds of particles or selectively focus the heavier ones by adjusting the voltages. Then the different concentration patterns of microbeads exhibited that the microspheres can be flexibly manipulated by changing the configurations of microheaters. Furthermore, for the first time, we achieved thermal-based continuous particle focusing in both conducting and insulating solutions using buoyancy convection, demonstrating that this method can be utilized to achieve both static and continuous particle manipulations in multiple liquid media. Finally, the feasibility of this device in effective wear measurement of machines was demonstrated by conducting systematic experiments of focusing nanocopper particles in the hydraulic oil. Therefore, this presented approach would be promising for a broad range of on-chip applications.

$M^{i}$ icrofluidic systems now have become increasingly attractive for microbeads synthesis, ${ }^{1,2}$ cell culture, ${ }^{3,4}$ and medical diagnostics ${ }^{5,6}$ because of their rapid and simple fabrication processes, low cost, and high sensitivity with small volumes of samples. ${ }^{7,8}$ Usually, microparticle concentration is one of the key steps in these microfluidic-based applications. Therefore, for facilitating the particle concentration in these applications, lots of passive methods ${ }^{9-11}$ and active ones ${ }^{12,13}$ have been studied. Specifically, the passive approaches do not consume external energy, but they are strictly restricted by the flow conditions and the geometry of the microchannels. ${ }^{14,15}$ Compared with the passive methods, the active ones only need simple channel structure and can be flexibly manipulated by external electric, ${ }^{16}$ acoustic, ${ }^{17}$ or magnetic field. ${ }^{18,19}$ For external-field-based manipulations, the electric field is convenient but limited by the highly strict solution conductivities and the easy electrode reactions. ${ }^{20,21}$ Acoustically controlled schemes are versatile, noninvasive, and flexible, but the acoustic generators are expensive and time-consuming for fabrication. $^{22,23}$ Although the magnetic force is useful and powerful for particle concentration, the applications are confined by the magnetism of microbeads and buffer solutions. $^{24,25}$

Recently, manipulation of microparticles by temperature gradient field has attracted tremendous attention for the simplicity of its manipulating mechanism. Temperature gradient in particle suspensions can cause thermal buoyancy convection, ${ }^{26}$ particle thermophoresis, ${ }^{27}$ and thermo-capillary convection. ${ }^{28}$ For buoyancy convection, it can generate threedimensional internal flow and form microvortices; the microparticles in granular fluid will migrate under the drag force of vortices. As for particle thermophoresis in liquid media, the thermophoretic ability is affected by many factors, such as particle size, ${ }^{29}$ temperature, ${ }^{30}$ and ion species. ${ }^{31}$ Under these effects, microbeads suspended in a temperature gradient field have the tendency to move toward the hot (negative thermophoresis) or cold (positive thermophoresis) region. $^{32-34}$ The thermo-capillary convection is caused by the

Received: November 5, 2018

Accepted: February 28, 2019

Published: February 28, 2019 
slip of unevenly heated gas-liquid (or liquid-liquid) interfaces. The three-dimensional convection and vortices are induced as the inner fluid will flow under the viscous effect of flowing interfaces. The microbeads in fluid then follow the motion of microvortices. ${ }^{35,36}$

On the basis of these three particle-manipulation mechanisms, thermal tweezers, exploiting highly focused lasers to generate temperature gradient in particle suspensions, have been widely used to separate, trap, and migrate particles. ${ }^{37,38}$ Although these existing optical methods based on temperature gradient are highly attractive for a broad range of on-chip applications, they have some disadvantages that are restricting their further applicabilities. First, these opto-thermal tweezers all require expensive and complicated laser systems and photosensitive substrates to heat the microfluids, which leads to a high-cost and complex experimental process. ${ }^{39,40}$ Second, most methods employ point-source lasers to manipulate microbeads, resulting in the particles being monotonously focused into circular clusters in the absence of extra manipulation strategies. Although some researchers achieved diverse concentration patterns by combining the complicated migration trajectories of laser beams and the complex configurations of photosensitive substrates, ${ }^{41,42}$ their methods were inconvenient for complex manipulations and integration of functions. To realize different manipulation objects and chip's functions, it is important to conveniently focus microparticles into different patterns. Last but not least, most methods utilize thermophoresis to achieve precise particle control. However, the thermophoretic velocity is usually very small $^{43,44}$ and typically requires a long migration time for microparticles. Although there are some opto-thermal tweezers based on thermal buoyancy convection or thermo-capillary convection, the former used very low channel height in which the buoyancy convection was restricted and very weak ${ }^{45}$ and the latter needed free surfaces, resulting in difficulty of continuous fluid injection. ${ }^{46}$ Therefore, these thermal tweezers usually serve for static manipulation. Obviously, they are lowthroughput and difficult for multistep processing of microspheres compared with the methods of particle manipulation in continuous flow. Regarding the limitations of these thermal tweezers, some researchers used combined temperatureelectric field to manipulate microbeads. ${ }^{47,48}$ Although the combined fields can improve the migration velocity, the electric field cannot be used to manipulate insulating solutions. Additionally, the highly focused lasers or microheaters made from multilayer materials in these devices are also complicated and expensive in the experimental process. Taking all these restrictions into consideration, we can find that the high cost, complexity, and low manipulation velocity of these devices are the main reasons confining their further applicabilities. Therefore, it is necessary for us to explore an alternative simpler and cheaper method to heat microfluids and to use new strategies to improve particle migration velocity.

In this Article, we propose a flexible and noninvasive approach for efficient concentration of microparticle in multiple liquid media based on direct current-induced thermal buoyancy convection (shown in Figure 1). This microdevice features simple and cheap indium tin oxide (ITO)-made microheaters to heat granular microfluids with a maximum consumed power of $<0.5 \mathrm{~W}$ (the electrode and heating mechanism are shown in parts $b$ and $c$ of Figure 1, respectively). For avoiding the possible electrochemical reactions on electrode, the electrode is isolated from
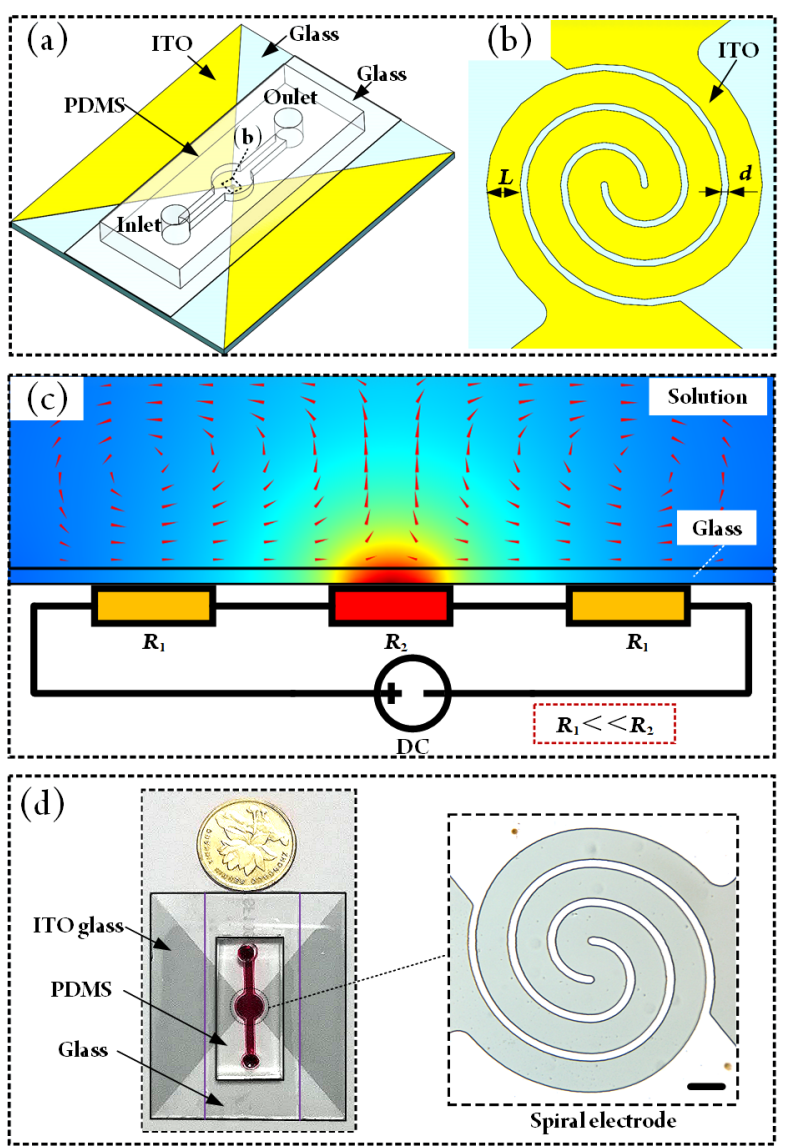

Figure 1. (a) Three-dimensional schematic of the trapping device. (b) Configuration of central spiral electrode. (c) Generation mechanism of thermal buoyancy convection: The fluid heated by spiral electrode becomes lighter and flows upward, then vortices (shown in (c)) are generated under the inducement of channel geometry. $R_{1}(3.5 \Omega)$ is the resistance of the extended region of ITO, and $R_{2}(93 \Omega)$ represents the resistance of the central spiral electrode. (d) Image of experimental chip. Scale bar, $100 \mu \mathrm{m}$.

microfluids by a $140-\mu \mathrm{m}$ thick glass slide. To improve the particle migration velocity, we use a $1.5 \mathrm{~mm}$ deep microchannel based on the effect of channel height on buoyancy convection. The different concentration patterns of microspheres can be conveniently achieved by adjusting the configurations of microheaters. The concentration performance of our chip was first demonstrated by statically focusing 4$\mu \mathrm{m}$ silica particles, yeast cells, silica particles in insulating organic buffer, and 100-nm copper microspheres. We also, for the first time, achieved thermal-based continuous particle focusing in multiple liquid media using thermal buoyancy convection. Lastly, the feasibility of this method in effective wear measurement of machines was demonstrated by conducting systematic experiments of focusing nanocopper particles in the hydraulic oil. This approach is attractive for microfluidic-based microparticle concentration.

\section{MATERIALS AND METHODS}

This microdevice contains three parts: a $1.1-\mathrm{mm}$ thick substrate glass with a $1-\mu \mathrm{m}$ thick indium tin oxide (ITO) film (South China Science \& Technology Company, China), a $140-\mu \mathrm{m}$ thick glass slide, and a polydimethylsiloxane (PDMS) microchannel. The electrode pattern is obtained through a standard etching process (the detailed process can be seen in 

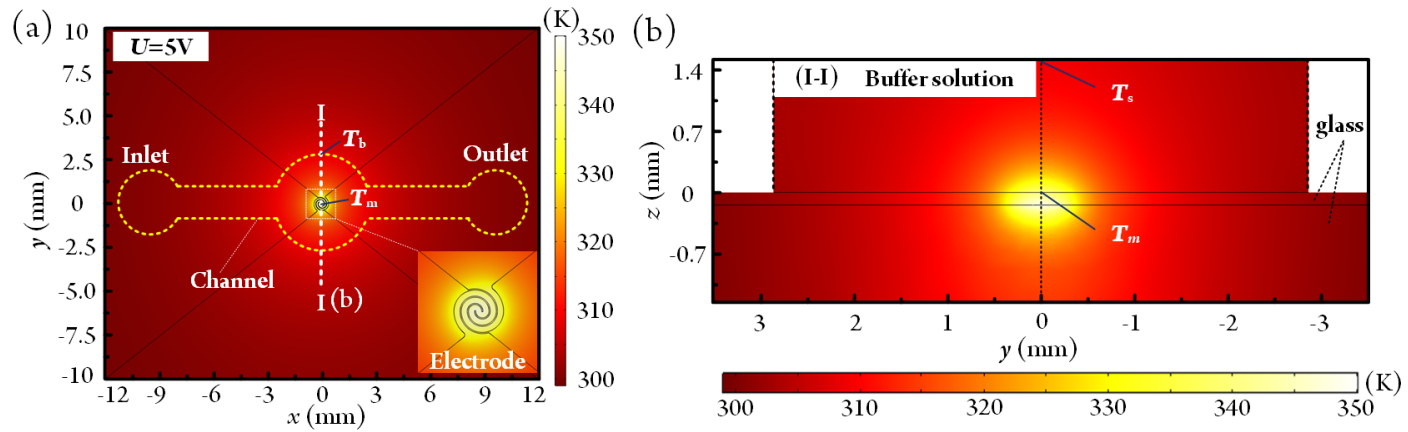

(c)

(d)
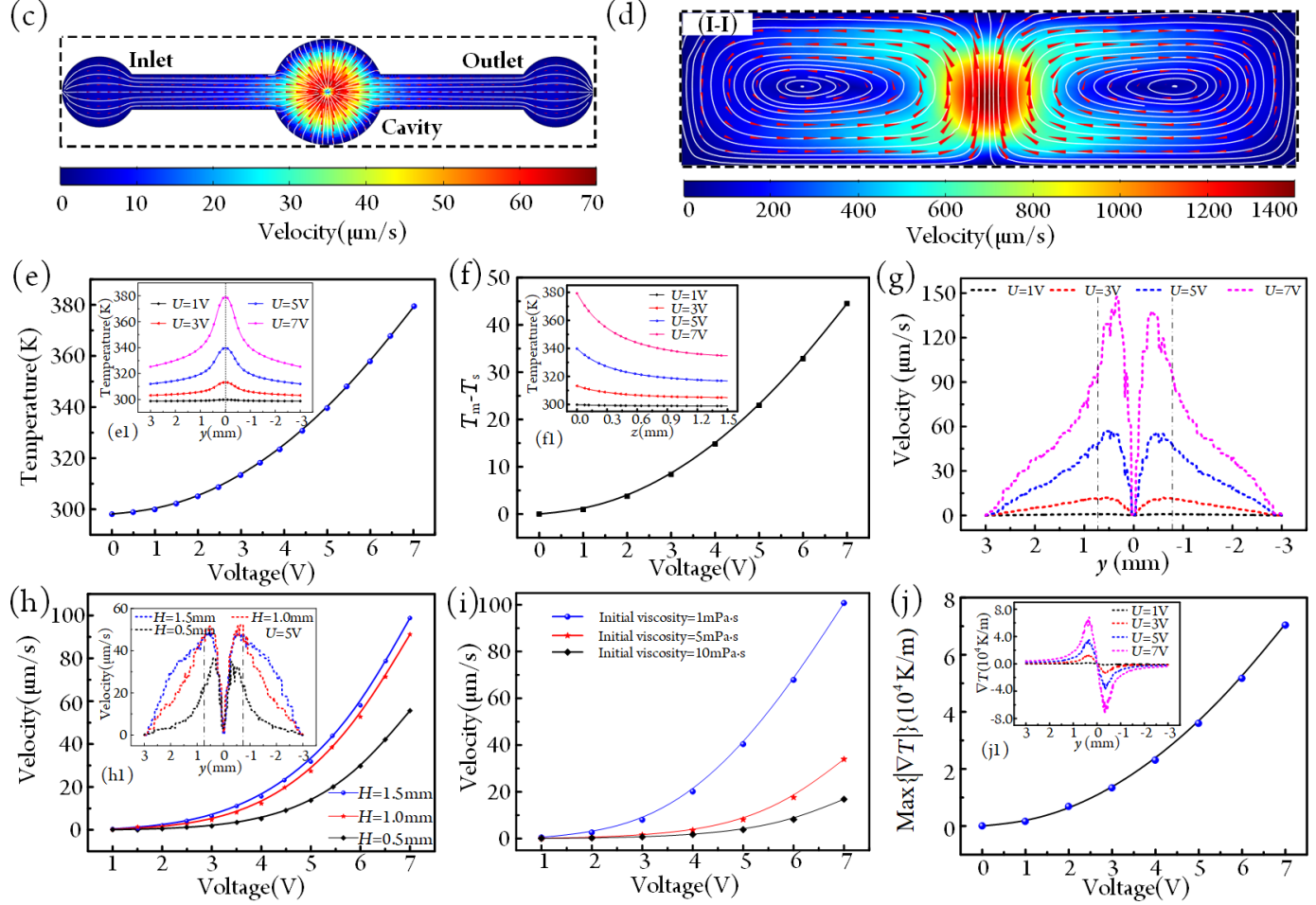

Figure 2. (a, b) Temperature distributions on the $x y$-plane at the fluid-wall interface (a) and $y z$-plane (b), actuated by a 5-V dc voltage. (c, d) Flow fields on the $x y$-plane when $z=10 \mu \mathrm{m}$ (c) and $y z$-plane (d). (e) Variation trend of the maximum temperature $T_{\mathrm{m}}$ under different dc voltages. (f) Temperature difference $T_{\mathrm{m}}-T_{\mathrm{s}}$ under different dc voltages. (g) Velocity distributions on I-I line (marked in Figure $1 \mathrm{a}$ ) when $z=10 \mu \mathrm{m}$. (h) Average flow rates on $\mathrm{I}-\mathrm{I}$ line $(z=10 \mu \mathrm{m})$ under different dc voltages and channel heights. (i) Average flow rates on $\mathrm{I}-\mathrm{I}$ line $(z=10 \mu \mathrm{m})$ under different dc voltages and fluid viscosities. ( $\mathrm{j}$ ) Maximum temperature differences on $\mathrm{I}-\mathrm{I}$ line at different dc voltages.

section $\mathrm{S} 1$ of the Supporting Information). As shown in Figure $1 \mathrm{~b}, L$ is $100 \mu \mathrm{m}$ and $d$ is $20 \mu \mathrm{m}$. The PDMS channel with a deepness of $1.5 \mathrm{~mm}$ is polymerized by using polymethylmethlacrylate (PMMA) or dry-film (RISTON SD238) molds. When these three parts are prepared, the thin glass slide is first bonded to the ITO glass surface using UV glue. Then the whole chip is assembled by aligning and bonding the PDMS channel and the thin glass slide together after an oxygen plasma treatment in a plasma cleaner (ZEPTO, Diener, Germany).

The particles and cells used in our study are $4-\mu \mathrm{m}$ silica particles (Sigma), polystyrene (PS) particles (Fluka) with two kinds of diameters ( 5 and $2 \mu \mathrm{m}), 100-\mathrm{nm}$ copper particles, yeast cells, and 4- $\mu \mathrm{m}$ PMMA microspheres (Sigma). The buffer solutions used to dilute the particle samples include deionized (DI) water, the mixture of DI water and glycerol, the mixture of glycerol and ethanol, and the hydraulic oil. The electrode is energized by a dc signal generator (KXN-305D, Zhao Xin). The experiments are observed by using a microscope (BX53, Olympus, Japan) with a charge-coupled device (CCD) camera (Retiga, 2000R, Qimaging). The temperature of the bottom center $T_{\mathrm{m}}$ (shown in Figure $3 \mathrm{~b}$ ) is measured by using a thermometer (TASI-8620).

\section{RESULTS AND DISCUSSION}

Numerical Analysis of Thermal Buoyancy Convection. On the basis of the numerical model in the Supporting Information, we established a three-dimensional simulation model using Comsol software (COMSOL Multiphysics 5.3) to analyze the buoyancy convection of water solution. Parts a and b of Figure 2 show the temperature distributions of the fluid excited by a spiral electrode at the bottom center. From the top view (Figure $2 a$ ) and side view (Figure $2 b$ ), the temperature is dissipated from the bottom center $\left(T_{\mathrm{m}}\right)$ to the bottom border $\left(T_{\mathrm{b}}\right)$ and from the bottom center $\left(T_{\mathrm{m}}\right)$ to the top center $\left(T_{\mathrm{s}}\right)$. The flow fields caused by temperature gradient are exhibited in Figure $2 \mathrm{c}$ and $\mathrm{d}$. From the top view (Figure 2c), all fluids flow toward the bottom center, and a flow stagnation region is formed here. Microparticles in buffer solution will follow the motion of fluid and be transferred to the stagnation region. 
(a)

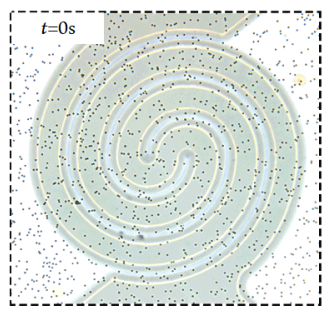

(b)
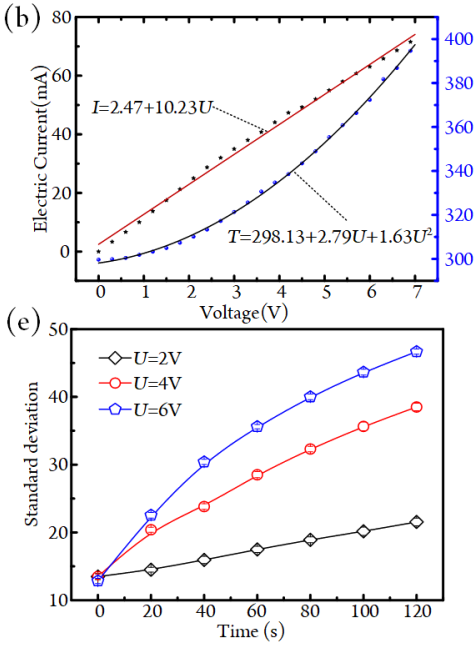

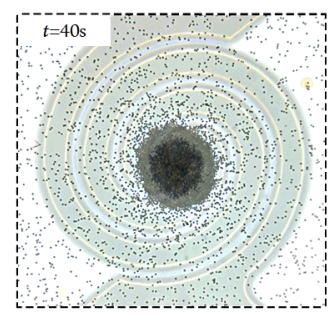

(c)

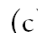

(f)

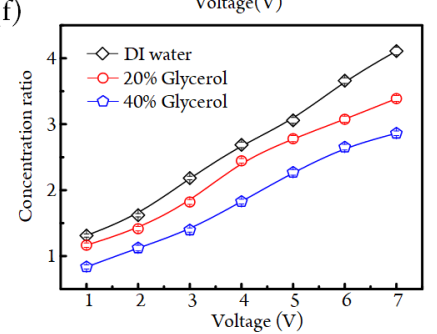

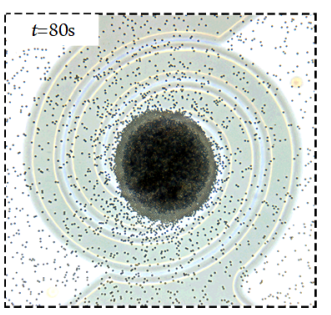

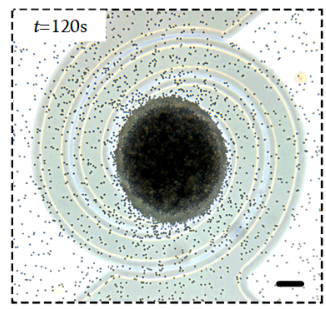

(d)

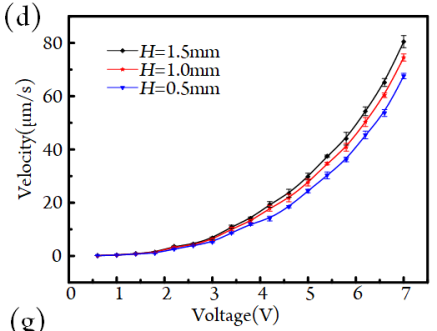

(g)

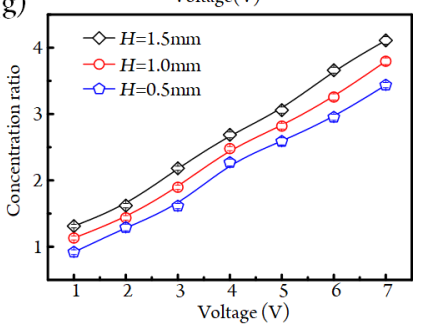

Figure 3. (a) Concentration profiles of silica particles under different time nodes at $U=5 \mathrm{~V}$ (see Movie S2). Scale bar, $100 \mu \mathrm{m}$. (b) Currentvoltage and temperature-voltage characteristics of the spiral electrode. (c) Aggregation rates of silica particles at the bottom center under different fluid viscosities. (d) Aggregation rates of silica particles at the bottom center under different channel heights. (e) Variation trends of the standard deviation of gray value at the electrode's surface under different dc voltages. (f) Concentration ratios of particle numbers under different fluid viscosities. (g) Concentration ratios of particle numbers under different channel heights.

Also, the simulations show a pair of counter-rotating microvortices on the side view (Figure 2d). Clearly, the bottom fluid first flows toward the bottom center and then vertically flows away from it. So the microparticles can be trapped at the bottom center if the downward gravity force acting on particles is greater than the upward fluidic drag force (the detailed force analysis is shown in Figure S1).

The temperature distributions on I-I line $(z=0 \mu \mathrm{m}$, and II line is marked in Figure 2a) at different voltages are given in Figure 2e1. We fitted a curve showing the variation trend of the maximum temperature $T_{\mathrm{m}}$ under different dc voltages. As shown in Figure 2e, $T_{\mathrm{m}}$ rises from room temperature (298.15 $\mathrm{K})$ to $380 \mathrm{~K}$ as the voltage increases from 0 to $7 \mathrm{~V}$. The vertical temperature distributions under different voltages are shown in Figure $2 \mathrm{f} 1$. Then we calculated the temperature difference $T_{\mathrm{m}}$ - $T_{s}$, which also increases with the rising $\mathrm{dc}$ voltages. Importantly, the $T_{\mathrm{m}}-T_{\mathrm{s}}$ can be as high as $44.5 \mathrm{~K}$ at a voltage of $7 \mathrm{~V}$ (shown in Figure 2f). As described in eq S9a, the volumetric buoyancy force is proportional to the temperature difference. Therefore, the thermal buoyancy convection will increase with the rise of dc voltage. Because the microparticles near the bottom cavity follow the motion of fluid, the flow rate of the bottom fluid contributes a lot to the aggregation rate of microbeads. Thereby, we exhibit the flow rate distributions on I-I line near the bottom of fluid under different voltages in Figure $2 \mathrm{~g}$. The maximum flow rate can reach $150 \mu \mathrm{m} / \mathrm{s}$ at $7 \mathrm{~V}$. Compared with the existing thermal tweezers, $^{45,49}$ the flow rate in our chip is enhanced at least by 1 order of magnitude.

Corresponding to the temperature distribution, buoyancy convection is characterized by a kind of three-dimensional internal flow, vertically flowing toward and away from the heat source center (Figure 2d). Because the magnitude of buoyancy force acting on the whole fluid ( $f_{\text {buoyancy }}$ ) in a microchannel satisfies $f_{\text {buoyancy }} \propto \rho_{\mathrm{m}} g \beta_{\mathrm{T}} L^{3} \Delta T$ (where $\rho_{\mathrm{m}}$ is the fluid density, $g$ is the gravitational acceleration, $\beta_{\mathrm{T}}$ is the thermal expansion coefficient of the fluid, $L$ is the characteristic size of the microchannel, and $\Delta T$ is the temperature difference), the convection flow caused by buoyancy force will also be affected by the geometry of the microchannel. Figure $2 \mathrm{~h}$ indicates that the channel height can apparently influence the intensity of induced microvortices. The velocity distributions on I-I line under different channel heights at $U=5 \mathrm{~V}$ are shown in Figure 2h1. As shown in Figure $2 \mathrm{~h}$, when the channel height is appropriately increased, the average flow rate of the bottom fluid can be significantly enhanced. This can be explained by the fact that the low channel height will restrain the volumetric vortices, resulting in the decrease of flow rate. Compared with the result in a $0.1-\mathrm{mm}$ deep microchannel, ${ }^{45}$ the thermal buoyancy convection is greatly improved in the $1.5-\mathrm{mm}$ deep microchannel. The flow rate is also influenced by the fluid viscosity (shown in Figure 2i). Specifically, the higher the fluid viscosity, the lower is the flow rate.

Apart from the fluidic drag force, at the presence of temperature gradient, suspended particles are also driven by the thermophoretic force. The thermophoretic velocity (eq $S 12$ ) is proportional to the thermal diffusion coefficient $D_{\mathrm{T}}$ and the temperature gradient $\nabla T$. As shown in Figure $2 \mathrm{j}$, the maximum value of $\nabla T$ is no more than $8 \times 10^{4} \mathrm{~K} / \mathrm{m}$. Also, the order of $D_{\mathrm{T}}$ is $\mu \mathrm{m}^{2} /(\mathrm{K} \mathrm{s}){ }^{31}$ Thus, the magnitude of thermophoretic velocity is no more than $1 \mu \mathrm{m} / \mathrm{s}$, which is far less than the buoyancy convection velocity (shown in 
Figure $2 \mathrm{~g}$ and $\mathrm{h}$ ). Therefore, the particle concentration rate is mainly related to the buoyancy convection in our chip. Importantly, to experimentally demonstrate the weak influence of thermophoresis on microparticle concentration, we utilize a microchannel with a height of $20 \mu \mathrm{m}$, in which the thermal buoyancy convection is very weak and therefore negligible, to investigate the thermophoretic behavior of $4-\mu \mathrm{m}$ silica particles. The result showed that the silica particles were almost static when the chip was energized at $3 \mathrm{~V}$ for $120 \mathrm{~s}$ (see Movie S1). However, the focusing speed of silica particles can reach $6.5 \mu \mathrm{m} / \mathrm{s}$ in a $1.5-\mathrm{mm}$ deep microchannel at the same voltage. Therefore, the effect of thermophoresis on particle motion is negligible.

Microsized Particle Concentration. As shown in Figure $2 c$, a single circular flow stagnation region will form at the bottom center of the fluid when we use the spiral electrode (shown in Figure 1) to heat the buffer solution. To demonstrate this method's feasibility in trapping microparticles, we first performed the concentration experiments of $4-\mu \mathrm{m}$ silica particles in water solution (DI water and the mixture of DI water and glycerol) (Figure 3). Figure 3a shows the concentration profiles of silica particles at different times. Specifically, under a dc voltage of $5 \mathrm{~V}$, a large amount of silica particles quickly focus to the bottom center (see Movie S2).

The experimental results showing the electric current and temperature as a function of the applied voltage for the spiral microheater are given in Figure $3 \mathrm{~b}$. According to the currentvoltage curve $(\mathrm{C}-\mathrm{V})$, the resistance of the ITO-made microheater is $\sim 100 \Omega$, and it does not vary with the temperature. The temperature-current curve $(\mathrm{T}-\mathrm{V})$ indicates that the temperature of the heat source center nonlinearly increases as the $\mathrm{dc}$ voltage rises. The comparison of $\mathrm{T}-\mathrm{V}$ curves in Figures $3 \mathrm{~b}$ and $2 \mathrm{e}$ can also demonstrate the veracity of our numerical analysis.

Just like the simulation results in Figure 2i, the fluid viscosity has a negative effect on the resulting fluid velocity. That is, the flow rate decreases from 100.7 to $16.8 \mu \mathrm{m} / \mathrm{s}$ as the fluid viscosity increases from 1 to $10 \mathrm{mPa} \cdot \mathrm{s}$ when the voltage is fixed at $7 \mathrm{~V}$. In our trapping method, the randomly distributed microparticles are driven to the flow stagnation region by the fluidic drag force (see section S2 of the Supporting Information), which is proportional to the fluid velocity. Thus, the aggregation rate of silica particles is negatively influenced by fluid viscosity (shown in Figure 3c). We studied the aggregation rate of silica particles in DI water, DI water containing $20 \%$ glycerol, and DI water containing $40 \%$ glycerol. It is found that, compared with the result in DI water, the concentration rate has declined by $32 \%$ in the solution containing $40 \%$ glycerol when the voltage is fixed at 7 V.

The simulation results in Figure $2 \mathrm{~h}$ indicate that the channel height also influences the motion characteristics of fluid. Thus, we implemented the concentration of silica particles (suspended in DI water) under three channel heights: 0.5, 1, and $1.5 \mathrm{~mm}$ (shown in Figure $3 \mathrm{~d}$ ). Figures $2 \mathrm{~h}$ and $3 \mathrm{~d}$ show good agreement with each other. That is, particle-aggregation rate can be improved by appropriately increasing the channel height. For example, when the voltage is $7 \mathrm{~V}$, the aggregation rate can be improved from 67.5 to $80.5 \mu \mathrm{m} / \mathrm{s}$ by rising the channel height from 0.5 to $1.5 \mathrm{~mm}$. More importantly, compared with the previous thermal tweezers, ${ }^{45}$ particleaggregation rate has been significantly improved in a $1.5-\mathrm{mm}$ deep microchannel.
Besides the particle-aggregation rate, the number of concentrated microparticles is also an important criterion evaluating the trapping efficiency. In microbeads concentration studies, two methods are commonly used to represent the particle numbers: one is the direct quantity statistics, and the other is the indirect gray-value distribution of concentration profiles. $^{50,51}$ In our experiments, it is difficult to count the particle numbers because of the large scale and highly effective concentration of microbeads (ss shown by the concentration profiles in Figure 3a). Thus, we use the second method to indirectly characterize the number of concentrated microparticles. When power is off, a small number of silica particles are evenly distributed at the electrode's surface (shown in Figure 3a). At this state, a minimum standard deviation of the gray value of the electrode's surface was obtained using ImageJ software (shown in Figure 3e). With the rising of time node and dc voltage, an increasing number of microbeads are trapped at the bottom center (shown in Figure 3a). After calculating the standard deviation of gray value of the electrode's surface under different time nodes and dc voltages, we found the same variation trend between the standard deviation of gray value and the number of concentrated particles (shown in Figure 3e). Therefore, it is feasible for us to use the standard deviation of gray value of the electrode's surface to indirectly evaluate the number of microspheres. Furthermore, we used the ratio of the standard deviation of gray value at $t=120 \mathrm{~s}$ to the initial standard deviation to represent the concentration ratio of particle numbers. Clearly, the concentration ratio increases with the rising voltages (shown in Figure $3 \mathrm{f}$ and $\mathrm{g}$ ). For example, the concentration ratio improves from 1.3 to 4.1 as the voltage rises from 1 to $7 \mathrm{~V}$ in DI water solution. In a buffer solution with higher viscosity, the aggregation rate and aggregated particles are remarkably decreased. Thus, the concentration ratio of particle numbers decreases with the increasing fluid viscosity (shown in Figure 3f). Similarly, because the channel height has a positive influence on the particle-aggregation rate (shown in Figure $3 \mathrm{~d})$, the concentration ratio increases as channel height rises (Figure 3g).

Additionally, we successfully conducted the efficient focusing of yeast cells, demonstrating that this method can be used to efficiently manipulate live cells (detailed information can be seen in section S4 of the Supporting Information). Moreover, for the first time, we carried out the concentration of particles in insulating organic buffer. There are few studies about the manipulation of insulating organic solution, so our study can extend the application fields (detailed information can be seen in section S5 of the Supporting Information).

Nanosized Particle Concentration. Apart from micronsized particles, nanosized particles are also commonly studied in the fields of chemistry, biology, and medicine, such as cell organelle, DNA, and proteins. To verify the ability of this method for focusing and manipulating nanoparticles, we concentrated $100-\mathrm{nm}$ copper particles (suspended in DI water). Compared with the micron-sized particles, the nanosized copper particles have a bigger diffusion coefficient $D=k_{\mathrm{B}} T / 6 \pi \mu r$ ( $k_{\mathrm{B}}$ is the Boltzmann constant, $T$ is the temperature, $\mu$ is the dynamic viscosity of fluid, and $r$ is the particle radius); their concentration distribution meets eqs S10-S15 (shown in section S2 of the Supporting Information). On the basis of this numerical model, we conducted time-dependent concentration simulation at $U=5 \mathrm{~V}$ by using 
Comsol software (shown in Figure $4 \mathrm{a}$ and $\mathrm{b}$ ). The initial concentration $C_{0}$ is set as $1 \mathrm{~mol} / \mathrm{m}^{3}$. The results show that the

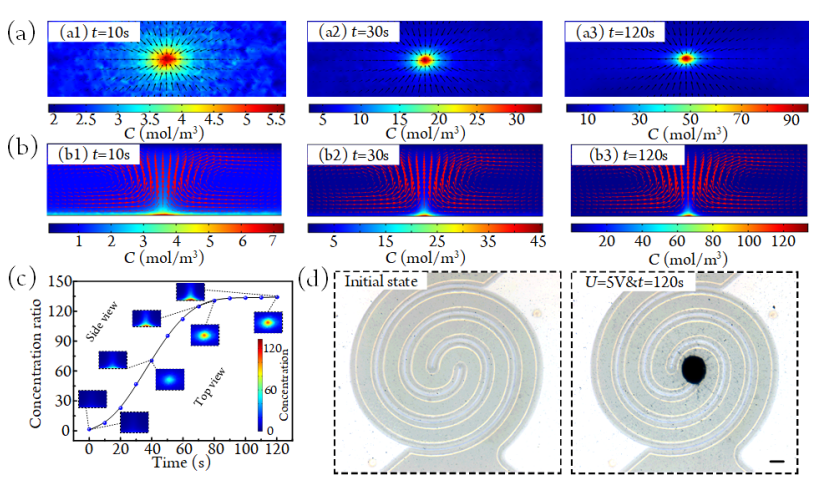

Figure 4. (a, b) Simulated concentration profiles of nanocopper particles under different time nodes: (a) top view and (b) side view. (c) Concentration ratio $\xi$ at different time nodes. (d) Trapping of 100-nm copper particles (see Movie S5). Scale bar, $100 \mu \mathrm{m}$.

concentration at the bottom center increases over time. Figure $4 \mathrm{c}$ shows the concentration ratio $\xi=C_{\max } / C_{0}$ under different time nodes. It reaches 120 at $t=120 \mathrm{~s}$, indicating an effective concentration performance. The experimental results are given in Figure 4d (see Movie S5). Before concentration, the 100-nm copper particles are difficult to find under a microscope at 40fold magnification (BX53, Olympus, Japan). When applying a $5-\mathrm{V}$ dc signal on the electrode for $120 \mathrm{~s}$, we can observe the accumulation of the copper particles into clusters at the bottom center. This experiment demonstrates that our particle concentration chip can be used to manipulate both microsized and nanosized microbeads.

Concentration of Particle Mixture. After the concentration of a single kind of particles, we further investigate the trapping of two kinds of particles (Figure 5; see Movie S6). Parts $\mathrm{b}-\mathrm{d}$ of Figure 5 show the concentration profiles of $5-\mu \mathrm{m}$ and $2-\mu \mathrm{m}$ PS beads under different voltages at $t=120 \mathrm{~s}$. As described in section S3 of the Supporting Information, the higher downward gravity force $\boldsymbol{F}_{\mathrm{G}}$ compared with the upward fluidic drag force $\boldsymbol{F}_{\text {drag }}\left(\boldsymbol{F}_{\mathrm{G}}>\boldsymbol{F}_{\text {drag }}\right)$ in the flow stagnation region is the main reason causing particle deposition and concentration. Under a low voltage $(<2 \mathrm{~V})$, two kinds of particles are both trapped at the bottom center (Figure $5 \mathrm{~b}$ and c). This phenomenon can be explained by the higher gravity forces acting on two kinds of particles compared with their fluidic drag forces. When the dc voltage is $>3 \mathrm{~V}$, there are only $5-\mu \mathrm{m}$ PS particles at the bottom center (Figure $5 \mathrm{~d}$ ). The reason is as follows: the gravity force of the $2-\mu \mathrm{m}$ PS beads is lower than the fluidic drag force, whereas the gravity force of the $5-\mu \mathrm{m}$ PS beads is still greater than the fluidic drag force. Figure 5e shows the percentage of two kinds of PS particles at the bottom center under different voltages. Specifically, the percentage of $2-\mu \mathrm{m}$ PS particles in the flow stagnation region decreases from $50 \%$ to $0 \%$ as the voltage rises from 0 to $4 \mathrm{~V}$. Aiming at particle mixtures, this experiment demonstrates that it is possible to trap particles with different sizes at a reasonably low voltage. When the voltage is appropriately increased, the heavier microbeads can be selectively trapped. Thus, this microfluidic platform has the potential to achieve particle separation.

Particle Concentration in Different Patterns. In microfluidic-based particle manipulations, apart from the single
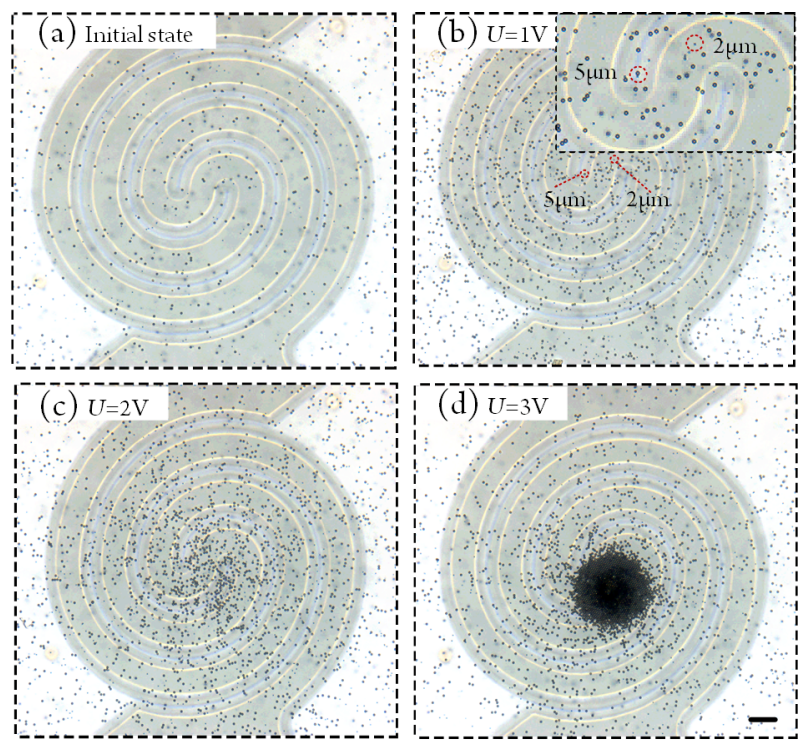

(e)

$5 \mu \mathrm{m}$ PS particles

\#2 $2 \mu \mathrm{m}$ PS particles

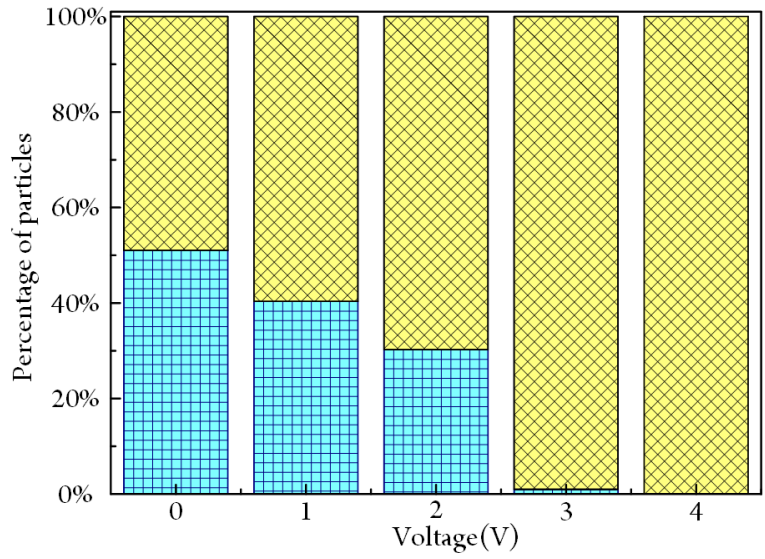

Figure 5. Trapping of two kinds of PS particles. (a) Initial state. (bd) Trapping profiles under different voltages (see Movie S6). Scale bar, $100 \mu \mathrm{m}$. (e) Percentage of PS particles under different voltages.

circular clusters, there are lots of occasions where the microbeads are supposed to focus into different patterns, such as the particle beam and multiclusters. The concentration patterns of microspheres are determined by the configurations of the flow stagnation region. In our method, the configuration of the flow stagnation region can be conveniently and cheaply adjusted by changing the patterns of ITO-made microheaters. To test our method's flexibility for forming diverse concentration patterns, besides the above single clustered concentration, we first focused the 4- $\mu \mathrm{m}$ PMMA particles into a single particle beam using a long strip-electrode to heat the buffer (Figure 6a; see Movie S7). As shown in Figure 6a, under a $7-\mathrm{V}$ dc voltage, the randomly distributed PMMA particles in a $190-\mu \mathrm{m}$ deep microchannel are focused to the center of the microheater within $10 \mathrm{~min}$. Additionally, we successfully conducted the trapping of PMMA particles at four circular flow stagnation regions under a $6-\mathrm{V}$ dc voltage (Figure $6 \mathrm{~b}$; see Movie S7). These two experiments show that the particlemanipulation platform has the potential to flexibly focus microparticles into complicated particle patterns.

Continuous Particle Focusing. Continuous focusing of particles into a narrow stream is usually necessary for upstream operation in numerous microfluidic applications such as 

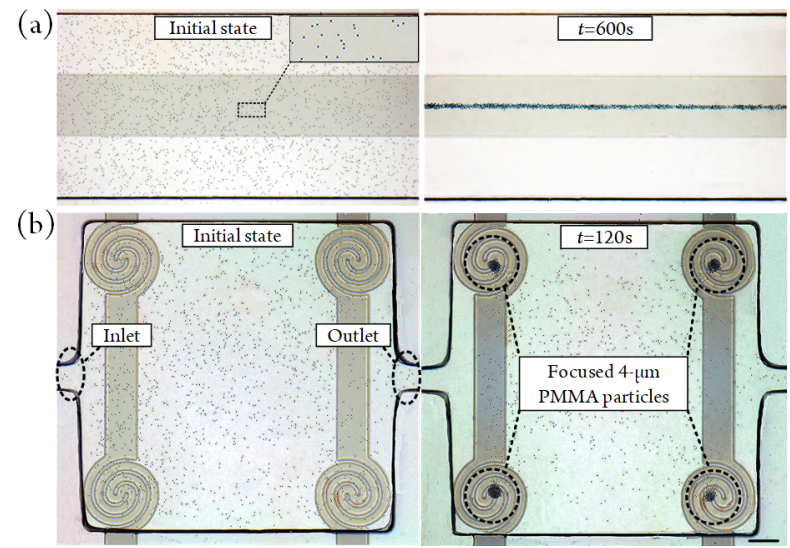

Figure 6. (a) Concentrated particle beam by using a long stripelectrode structure. (b) Simultaneous microbeads focusing at four circular flow stagnation regions (see Movie S7). Scale bar, $200 \mu \mathrm{m}$.

fluorescence-activated cell sorting (FACS). Thus, it is meaningful to realize continuous thermal-based particle focusing. However, as discussed in the first section, the current thermal tweezers are all low-throughput static particleconcentration strategies. Therefore, a further attempt was made to conduct continuous particle concentration by using our particle-manipulation platform. First, we conducted the continuous focusing of 4- $\mu \mathrm{m}$ PMMA particles in water solution at $U=7 \mathrm{~V}$ and $Q_{\mathrm{v}}=500 \mathrm{~nL} / \mathrm{s}$. As shown in Figure $7 \mathrm{a}$, apart

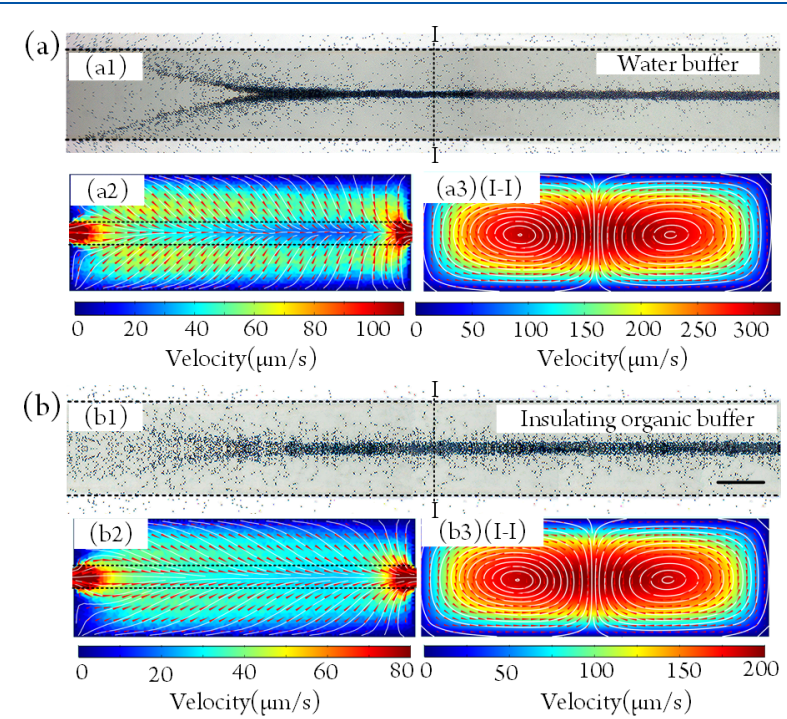

Figure 7. (a) Focusing of 4- $\mu \mathrm{m}$ PMMA particles in water solution at $U=7 \mathrm{~V}$ and $Q_{\mathrm{v}}=500 \mathrm{~nL} / \mathrm{s}$. (a1) Experimental image of PMMA particle beam. (a2, a3) Flow fields on the top view and side view. (b) Focusing of $4-\mu \mathrm{m}$ PMMA particles in absolute ethanol containing $50 \%$ glycerol at the same voltage and flow rate (see Movie S8). Scale bar, $250 \mu \mathrm{m}$. (b1) Experimental image. (b2, b3) Flow fields.

from axial migration, microparticles in water solution are also driven to the bottom center by counter-rotating microvortices. The microparticles are gradually concentrated into a continuous particle beam by the effect of continuous transverse vortices. Then the trapping experiments of PMMA particles in absolute ethanol containing $50 \%$ glycerol were implemented at the same voltage and flow rate (Figure $7 \mathrm{~b}$ ). According to eq S9a (shown in section S2 of the Supporting Information), the thermal expansion coefficient of buffer solution has a positive influence on buoyancy convection. The thermal expansion coefficients of the water, glycerol, and absolute ethanol are 2.08 $\times 10^{-4} / \mathrm{K}, 5 \times 10^{-4} / \mathrm{K}$, and $1 \times 10^{-3} / \mathrm{K}$, respectively. Thus, the thermal expansion coefficient of absolute ethanol containing $50 \%$ glycerol in our simulation was set as $\sim 7.5 \times 10^{-4} / \mathrm{K}$. Also, the initial viscosity of ethanol containing $50 \%$ glycerol is $\sim 5$ times greater than that of water solution. After setting different thermal expansion coefficients and the initial fluid viscosities, we conducted the numerical analyses of the buoyancy convection in these two kinds of liquid media. The results in Figure $7 \mathrm{a} 3$ and $\mathrm{b} 3$ show that the convection velocity in insulating organic solution is $\sim 1.5$ times smaller than that in water buffer, which also can be demonstrated by the experimental videos shown in Movie S8. The continuous focusing of PMMA particles in both conducting and insulating fluids demonstrates that this method can be used to continuously manipulate microbeads suspending in multiple liquid media. More importantly, compared with the current static thermal-based tweezers, this microdevice for continuous particle manipulation has a higher throughput and can integrate with other functional units to achieve multistep processing of microbeads.

Metallic Nanoparticle Focusing in Hydraulic Oil. In the machinery industry, $>75 \%$ of mechanical failures are wear failure. $^{52}$ The micro/nanosized metallic wear debris in lubricating and hydraulic oil carry a lot of information about the working condition of machines. Therefore, the real-time monitoring and analysis of these microparticles in machine oil can timely and accurately predict equipment failures. Also, the information carried by these particles can guide scientific lubrication, an efficient method to reduce equipment failure probabilities. In the early wear stage of machines, the number of metallic nanoparticles in machine oil is very small, resulting in a difficult observation and analysis process. For facilitating the monitoring of metallic particles in hydraulic oil, it is necessary to first achieve the particle prefocusing. To demonstrate this method's feasibility in the application of oil monitoring, we conducted the focusing of 100-nm copper particles in a highly viscous hydraulic oil with a viscosity of $43.94 \mathrm{mP} \cdot \mathrm{s}$ at $40^{\circ} \mathrm{C}$ (Total Hydransafe HFDU 46; the detailed parameters are shown in Table S1). When the voltage is fixed at $6 \mathrm{~V}$, Figure $8 \mathrm{a}$ exhibits the concentration profiles at different time nodes (see Movie S9). Clearly, the number of particles focused in the bottom center increases over time. Figure $8 \mathrm{~b}$ shows the enriching states of granular fluids with three particle concentrations $(0.25,0.5$, and $1 \mathrm{mg} / \mathrm{mL})$ at $t=30 \mathrm{~min}$ and $U$ $=6 \mathrm{~V}$. Specifically, the number of concentrated particles increases as the particle concentration rises. The number of particles in granular fluids is an important data to indicate the wear condition of machines. To quantitatively evaluate the particle number, we used the gray-value distribution at the electrode's surface to indirectly indicate the number of focused particles. Figure $8 \mathrm{c}$ shows the gray-value distribution at $t=30$ $\min , U=6 \mathrm{~V}$, and $C=0.25 \mathrm{mg} / \mathrm{mL}$. According to the standard deviations of gray value at different time points, we obtained the variation trends of standard deviation at different particle concentrations. As shown in Figure 8d, the slope of standard deviation increases with the rising particle concentrations, and the value of standard deviation gradually increases over time. The slope of the standard deviation-time curve can be used to compare the particle concentration speed at three concentrations, and the value of standard deviation indicates the relative numbers of trapped particles. 

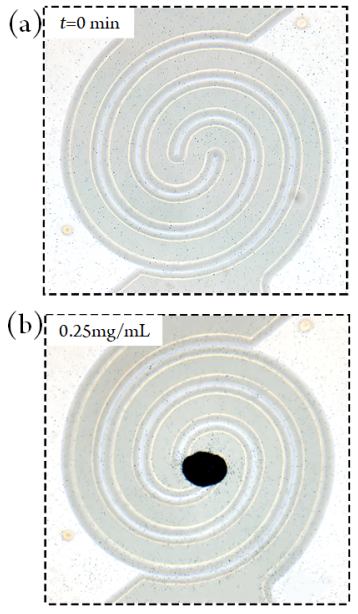
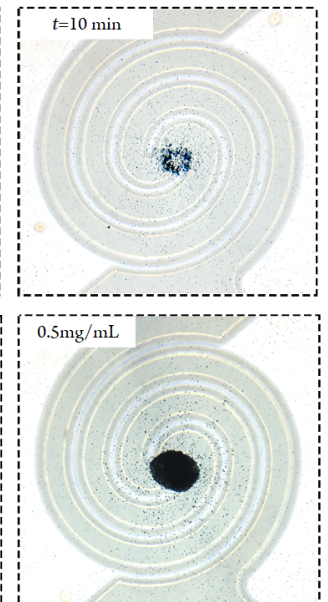
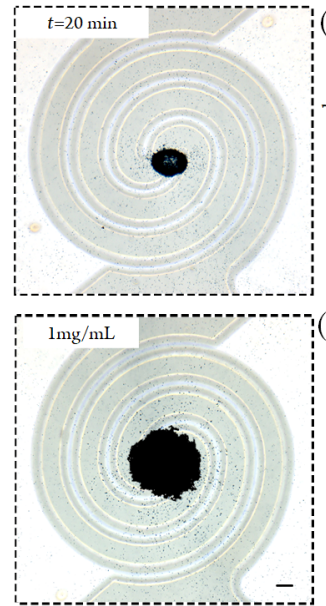

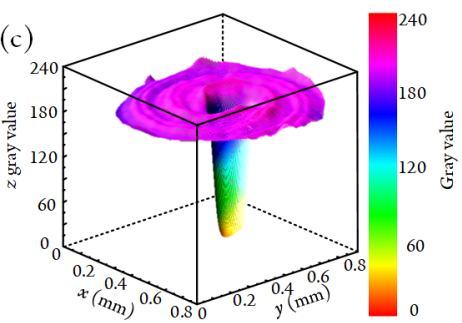

(d)

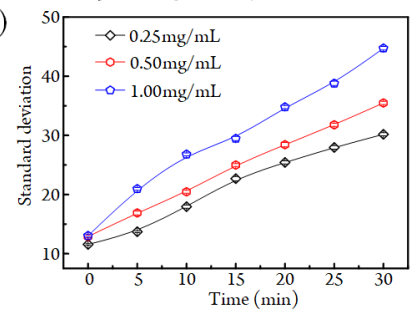

Figure 8. (a) Concentration profiles of nanocopper particles in hydraulic oil at different time nodes when dc voltage is fixed at $6 \mathrm{~V}$ (see Movie S9). (b) Concentration states of granular fluids with three concentrations at $t=30 \mathrm{~min}$ and $U=6 \mathrm{~V}$. Scale bar, $100 \mu \mathrm{m}$. (c) Gray-value distribution of the electrode's surface at $t=30 \mathrm{~min}, U=6 \mathrm{~V}$, and $C=0.25 \mathrm{mg} / \mathrm{mL}$. (d) Standard deviations of gray value at different time nodes and particle concentrations.

\section{CONCLUSIONS}

We developed a novel approach for efficient particle concentration in multiple liquid media by direct currentinduced thermal buoyancy convection. The influences of dc voltage, channel height, and fluid viscosity on the chip's trapping characteristics were first analyzed by concentrating 4$\mu \mathrm{m}$ silica particles. Specifically, the increased dc voltage and channel height positively influence the particle-aggregation rate and the concentration ratio of particle numbers, while the fluid viscosity has an opposite effect. The aggregation rate (concentration ratio) increases from $0(1.3)$ to $80.5 \mu \mathrm{m} / \mathrm{s}$ (4.1) in DI water as the voltage rises from 0 to $7 \mathrm{~V}$. Also, when the voltage is fixed at $7 \mathrm{~V}$, the aggregation rate (concentration ratio) can be improved from 67.5 (3.44) to $80.5 \mu \mathrm{m} / \mathrm{s}$ (4.11) by changing the channel height from 0.5 to $1.5 \mathrm{~mm}$. As for the influence of fluid viscosity, compared with the result in DI water, the flow rate (concentration ratio) decreases by $32 \%$ (30.4\%) in DI water containing $40 \%$ glycerol. The flexible particle concentration ability of this device was also demonstrated by trapping yeast cells, silica particles in insulating organic buffer, and 100-nm copper particles. The trapping experiments of $5-\mu \mathrm{m}$ and $2-\mu \mathrm{m}$ PS microbeads indicated that the chip can either simultaneously concentrate two kinds of particles when the voltage is no more than $2 \mathrm{~V}$ or selectively focus the heavier ones when the voltage is $>3 \mathrm{~V}$. The different concentration patterns of microbeads demonstrated that we can flexibly and cheaply adjust the trapping profiles by changing the configurations of microheaters. Furthermore, for the first time, we achieved continuous particle focusing in both conducting and insulating buffer solutions using buoyancy convection. As a consequence, this thermal-based particle focusing method can be used to achieve both static and continuous particle manipulations in multiple liquid media. Finally, the feasibility of this method in effective wear measurement of machines was demonstrated by conducting systematical experiments of nanocopper particle concentration within the hydraulic oil. Therefore, this presented approach can be attractive for a broad range of applications for its low cost, simplicity, flexibility, high efficiency, and noncontact with buffer solution.

\section{ASSOCIATED CONTENT}

\section{(S) Supporting Information}

The Supporting Information is available free of charge on the ACS Publications website at DOI: 10.1021/acs.analchem.8b05105.

Fabrication process of the ITO-made microheater, theoretical background, force analysis acting on particles in the flow stagnation region, enriching of yeast cells, enriching of silica particle in insulating organic buffer, and characteristics of hydraulic oil used in our experiment (PDF)

Thermophoretic behavior of 4- $\mu \mathrm{m}$ silica particles (AVI) Enriching of $4-\mu \mathrm{m}$ silica particles under diverse $\mathrm{dc}$ voltages (AVI)

Enriching of yeast cells (AVI)

Enriching of 4- $\mu \mathrm{m}$ silica particles in insulating organic solution (AVI)

Enriching of 100-nm copper particles (AVI)

Concentration of the mixture of $2-\mu \mathrm{m}$ and $5-\mu \mathrm{m}$ PS microspheres (AVI)

Microparticle concentration in different patterns (AVI) Continuous particle focusing via thermal buoyancy convection (AVI)

Metallic nanoparticle focusing in hydraulic oil (AVI)

\section{AUTHOR INFORMATION}

\section{Corresponding Authors}

*E-mail: rykhit@hit.edu.cn. Phone: +86 451 86418028. Fax: +8645186402658.

*E-mail: jhy_hit@hit.edu.cn. Phone: +86 451 86418028. Fax: +8645186402658.

\section{ORCID}

Yukun Ren: 0000-0002-0167-1274

Notes

The authors declare no competing financial interest.

\section{ACKNOWLEDGMENTS}

This work is financially supported by the National Natural Science Foundation of China (nos. 11672095, 11872165, 11702075, and 11702035) , Self-Planned Task 
(SKLRS201803B) of State Key Laboratory of Robotics and System (HIT), and Opening Fund of State Key Laboratory of Nonlinear Mechanics. We are also grateful to Yankai Jia and Xiangsong Feng for their assistance in the results discussions.

\section{REFERENCES}

(1) Yang, S.; Luo, X. Nanoscale 2014, 6, 4438-4457.

(2) Chiu, C. Y.; Yang, M. Y.; Lin, F. C.; Huang, J. S.; Huang, M. H. Nanoscale 2014, 6, 7656-7665.

(3) Avesar, J.; Blinder, Y.; Aktin, H.; Szklanny, A.; Rosenfeld, D.; Savir, Y.; Bercovici, M.; Levenberg, S. Anal. Chem. 2018, 90, 74807488.

(4) Zhao, S. P.; Ma, Y.; Lou, Q.; Zhu, H.; Yang, B.; Fang, Q. Anal. Chem. 2017, 89, 10153-10157.

(5) Abd Rahman, N.; Ibrahim, F.; Yafouz, B. Sensors 2017, 17, 449.

(6) Adekanmbi, E. O.; Srivastava, S. K. Lab Chip 2016, 16, 21482167.

(7) Li, S.; Cui, H.; Yuan, Q.; Wu, J.; Wadhwa, A.; Eda, S.; Jiang, H. Biosens. Bioelectron. 2014, 51, 437-443.

(8) Thorsen, T.; Maerkl, S. J.; Quake, S. R. Science 2002, 298, 580584.

(9) Chen, Q.; Li, D.; Lin, J.; Wang, M.; Xuan, X. Anal. Chem. 2017, $89,6915-6920$

(10) Lu, X.; Xuan, X. Anal. Chem. 2015, 87, 4560-4565.

(11) Liu, C.; Xue, C.; Chen, X.; Shan, L.; Tian, Y.; Hu, G. Anal. Chem. 2015, 87, 6041-6048.

(12) Ouyang, W.; Ye, X.; Li, Z.; Han, J. Nanoscale 2018, 10, 1518715194.

(13) Ouyang, W.; Li, Z.; Han, J. Anal. Chem. 2018, 90, 11366.

(14) Kim, J. A.; Lee, J. R.; Je, T. J.; Jeon, E. C.; Lee, W. Anal. Chem. 2018, 90, 1827-1835.

(15) Yang, S. H.; Lee, D. J.; Youn, J. R.; Song, Y. S. Anal. Chem. 2017, 89, 3639-3647.

(16) Chen, X.; Ren, Y.; Liu, W.; Feng, X.; Jia, Y.; Tao, Y.; Jiang, H. Anal. Chem. 2017, 89, 9583-9592.

(17) Destgeer, G.; Ha, B. H.; Park, J.; Jung, J. H.; Alazzam, A.; Sung, H. J. Anal. Chem. 2015, 87, 4627-4632.

(18) Lim, J.; Lanni, C.; Evarts, E. R.; Lanni, F.; Tilton, R. D.; Majetich, S. A. ACS Nano 2011, 5, 217-226.

(19) Chen, Q.; Li, D.; Malekanfard, A.; Cao, Q.; Lin, J.; Wang, M.; Han, X.; Xuan, X. Anal. Chem. 2018, 90, 8600-8606.

(20) Ren, Y.; Liu, X.; Liu, W.; Tao, Y.; Jia, Y.; Hou, L.; Li, W.; Jiang, H. Electrophoresis 2018, 39, 597-607.

(21) Ren, Y.; Liu, W.; Wang, Z.; Tao, Y. Phys. Fluids 2018, 30, No. 062006.

(22) Collins, D. J.; Ma, Z.; Ai, Y. Anal. Chem. 2016, 88, 5513-5522.

(23) Ding, X.; Li, P.; Lin, S. C.; Stratton, Z. S.; Nama, N.; Guo, F.; Slotcavage, D.; Mao, X.; Shi, J.; Costanzo, F.; Huang, T. J. Lab Chip 2013, 13, 3626-3649.

(24) van Reenen, A.; de Jong, A. M.; den Toonder, J. M.; Prins, M. W. Lab Chip 2014, 14, 1966-1986.

(25) Rodriguez-Villarreal, A. I.; Tarn, M. D.; Madden, L. A.; Lutz, J. B.; Greenman, J.; Samitier, J.; Pamme, N. Lab Chip 2011, 11, 12401248.

(26) Zheng, J.; Xing, X.; Yang, J.; Shi, K.; He, S. NPG Asia Mater. 2018, 10, 340-351.

(27) Dong, R.-Y.; Zhou, Y.; Yang, C.; Cao, B.-Y. Microfluid. Nanofluid. 2015, 19, 805-811.

(28) Winterer, F.; Maier, C. M.; Pernpeintner, C.; Lohmuller, T. Soft Matter 2018, 14, 628-634.

(29) Putnam, S. A.; Cahill, D. G.; Wong, G. C. Langmuir 2007, 23, 9221-9228.

(30) Piazza, R.; Parola, A. J. Phys.: Condens. Matter 2008, 20, 153102.

(31) Wurger, A. Phys. Rev. Lett. 2008, 101, 108302.

(32) Yan, Z.; Huang, X.; Yang, C. Microfluid. Nanofluid. 2015, 18, 403-414.
(33) Zhao, Y.; Zhao, C.; He, J.; Zhou, Y.; Yang, C. Soft Matter 2013, 9, 7726 .

(34) Shameli, S. M.; Ren, C. L. Anal. Chem. 2015, 87, 3593-3597.

(35) Muñoz, E.; Quispe, J.; Lambert, P.; Bolopion, A.; Terrazas, R.; Régnier, S.; Vela, E. Journal of Micro-Bio Robotics 2017, 12, 65-72.

(36) Quispe, J.; Muñoz, E.; Vela, E. Appl. Phys. Lett. 2016, 109, 124102.

(37) Yu, L. H.; Chen, Y. F. Anal. Chem. 2015, 87, 2845-2851.

(38) Xin, H.; Bao, D.; Zhong, F.; Li, B. Laser Phys. Lett. 2013, 10, No. 036004

(39) Zhang, S.; Juvert, J.; Cooper, J. M.; Neale, S. L. Sci. Rep. 2016, 6, 32840.

(40) Yang, Y.; Mao, Y.; Shin, K. S.; Chui, C. O.; Chiou, P. Y. Sci. Rep. 2016, 6, 22630.

(41) Peng, X.; Lin, L.; Hill, E. H.; Kunal, P.; Humphrey, S. M.; Zheng, Y. J. Phys. Chem. C 2018, 122, 24226.

(42) Ota, S.; Wang, S.; Wang, Y.; Yin, X.; Zhang, X. Nano Lett. 2013, $13,2766-2770$

(43) Xin, H.; Lei, H.; Zhang, Y.; Li, X.; Li, B. Opt. Express 2011, 19, $2711-2719$.

(44) Lei, H.; Zhang, Y.; Li, B. Opt. Express 2012, 20, 1292-1300.

(45) Flores-Flores, E.; Torres-Hurtado, S. A.; Paez, R.; Ruiz, U.; Beltran-Perez, G.; Neale, S. L.; Ramirez-San-Juan, J. C.; RamosGarcia, R. Biomed. Opt. Express 2015, 6, 4079-4087.

(46) Pradhan, T. K.; Panigrahi, P. K. Exp. Fluids 2015, 56. DOI: $10.1007 / \mathrm{s} 00348-015-2051-2$

(47) Chen, Y. L.; Yang, C. X.; Jiang, H. R. Sci. Rep. 2018, 8, 5945. (48) Velasco, V.; Williams, S. J. J. Colloid Interface Sci. 2013, 394, 598-603.

(49) Lei, H.; Zhang, Y.; Li, X.; Li, B. Lab Chip 2011, 11, 2241-2246.

(50) Zhu, G. P.; Hejiazan, M.; Huang, X.; Nguyen, N. T. Lab Chip 2014, 14, 4609-4615.

(51) Hammarstrom, B.; Laurell, T.; Nilsson, J. Lab Chip 2012, 12, $4296-4304$

(52) Yao, C. J.; Chen, X. H. Ordnance Industry Automation 2009, 28, $56-57$. 